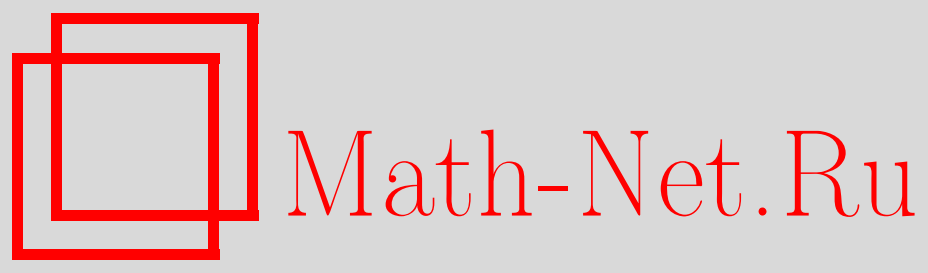

С. М. Гусейн-Заде, Ф. Дельгадо, А. Кампильо, Многочлены Александера и ряды Пуанкаре наборов идеалов, Функи. анализ и его прил., 2011, том 45, выпуск 4, 40-48

DOI: https://doi.org/10.4213/faa3043

Использование Общероссийского математического портала MathNet.Ru подразумевает, что вы прочитали и согласны с пользовательским соглашением

http://www . mathnet.ru/rus/agreement

Параметры загрузки:

IP : 3.85 .183 .62

26 апреля 2023 г., 14:35:44

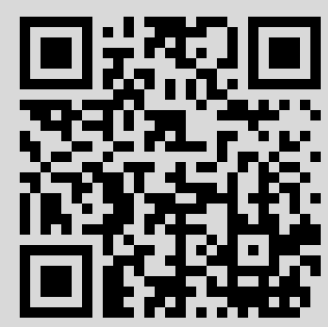




\title{
Многочлены Александера и ряды Пуанкаре наборов идеалов*
}

\author{
(c) 2011. С. М. ГУСЕЙН-ЗАДЕ, Ф. ДЕЛГАДО, А. КАМПИЛЬО
}

\section{Памяти В. И. Арнолъда}

\begin{abstract}
Ранее авторы рассмотрели и в некоторых случаях вычислили ряды Пуанкаре двух типов мультииндексных фильтраций на кольце ростков функций на (нормальной) комплексной особенности поверхности. Фильтрация первого типа определялась кривой (с несколькими компонентами) на особенности поверхности. Другая (так называемая дивизориальная фильтрация) определялась набором компонент исключительного дивизора модификации особенности поверхности. Здесь определяется и в некоторых случаях вычисляется ряд Пуанкаре, соответствующий набору идеалов в кольце ростков функций на особенности поверхности. Для комплексной плоскости это понятие объединяет два описанных выше типа фильтраций.
\end{abstract}

В [2], [8], [4], [5] были рассмотрены и в некоторых случаях вычислены ряды Пуанкаре двух типов мультииндексных фильтраций на кольце ростков функций на комплексной (нормальной) особенности поверхности $(S, 0)$ (в частности на $\left.\left(\mathbb{C}^{2}, 0\right)\right)$. Фильтрация первого типа определялась кривой (с несколькими компонентами) на $(S, 0)$. Для $(S, 0)=\left(\mathbb{C}^{2}, 0\right)$ соответствующий ряд Пуанкаре оказался совпадающим с многочленом Александера (от нескольких переменных) соответствующего (алгебраического) зацепления (когда число компонент кривой больше 1; иначе он равен соответствующей дзета-функции, т. е. многочлену Александера, деленному на $1-t)$. Отождествление всех переменных приводит к дзета-функции монодромии особенности кривой. Фильтрация второго типа (так называемая дивизориальная) определялась набором компонент исключительного дивизора некоторой модификации особенности поверхности $(S, 0)$. Здесь мы определяем понятие ряда Пуанкаре набора идеалов в кольце $\mathcal{O}_{(S, 0)}$ ростков функций на $(S, 0)$, описываем его связь с рядами Пуанкаре фильтраций и в некоторых случаях вычисляем ряды Пуанкаре наборов идеалов.

Обсуждаемое понятие ряда Пуанкаре было навеяно понятием дзета-функции идеала, сформулированным в [14]. Для наших целей удобно распространить это понятие на конечный набор идеалов $\left\{I_{1}, \ldots, I_{r}\right\}$, определив «многочлен Александера» этого набора. Это «смесь» понятий, введенных в [14] и [15].

Пусть $(V, 0)$ - росток аналитического пространства с изолированной особой точкой в начале координат, и пусть $I_{1}, \ldots, I_{r}-$ идеалы в кольце $\mathcal{O}_{V, 0}$ ростков функций на $(V, 0)$. Пусть $\pi:(X, D) \rightarrow(V, 0)$ - разрешение особенности пространства $V$, а также набора идеалов $\left\{I_{1}, \ldots, I_{r}\right\}$. Это означает, что:

(i) $X$ - комплексно-аналитическое многообразие;

*Работа первого автора частично поддержана грантами РФФИ-10-01-00678 и НШ8462.2010.1. Второй и третий авторы частично поддержаны грантом МТМ2007-64704. 
(ii) $\pi$ - собственное аналитическое отображение, являющееся изоморфизмом вне объединения множеств нулей идеалов $I_{1}, \ldots, I_{r}$;

(iii) исключительный дивизор $D=\pi^{-1}(0)$ является дивизором с нормальными пересечениями на $X$;

(iv) для $i=1, \ldots, r$ поднятие $I_{i}^{*}=\pi^{*} I_{i}$ идеала $I_{i}$ на пространство разрешения $X$ является локально главным (и поэтому $\pi$ является «принципализацией» идеала $\left.I_{i}\right)$;

(v) объединение множеств нулей поднятий идеалов $I_{1}, \ldots, I_{r}$ на пространство разрешения $X$ является дивизором с нормальными пересечениями на $X$.

Для $\underline{k}=\left(k_{1}, \ldots, k_{r}\right) \in \mathbb{Z}_{\geqslant 0}^{r}$ пусть $S_{\underline{k}}$ - множество точек $x \in D$, таких, что в некоторых локальных координатах $z_{1}, \ldots, z_{n}$ на $X$ с началом в точке $x$ множество нулей идеала $I_{i x}^{*}, i=1, \ldots, r$, является гиперповерхностью $\left\{z_{1}=0\right\}$ с кратностью $k_{i}$, т. е. $I_{i x}^{*}=\left\langle z_{1}^{k_{i}}\right\rangle$.

Определение. Многочлен Александера набора идеалов $\left\{I_{i}\right\}$ - рациональная функция (или степенной ряд) от переменных $t_{1}, \ldots, t_{r}$, задаваемая формулой типа А'Кампо [1]:

$$
\Delta_{\left\{I_{i}\right\}}\left(t_{1}, \ldots, t_{r}\right)=\prod_{\underline{k} \in \mathbb{Z}_{\geqslant 0}^{r} \backslash\{0\}}(1-\underline{t} \underline{k})^{-\chi\left(S_{\underline{k}}\right)},
$$

где $\chi(\cdot)$ - эйлерова характеристика, а $\underline{t} \underline{k}:=t_{1}^{k_{1}} \cdots t_{r}^{k_{r}}$.

Замечания. 1. Для $r=1$ это дает понятие дзета-функции $\zeta_{I}(t)$ идеала $I$ (см. [14]). В [14, теорема 4.2] забыли написать, что отображение $\pi$ должно быть изоморфизмом вне множества нулей идеала $I$. Из-за этого мы формулируем понятие многочлена Александера набора идеалов для ростков $(V, 0)$ с изолированными особенностями. Другой возможностью является требование, чтобы множество особых точек пространства $V$ содержалось в множестве нулей идеала $I$.

2. Многочлен Александера $\Delta_{\left\{I_{i}\right\}}\left(t_{1}, \ldots, t_{r}\right)$, определенный таким образом, вообще говоря, многочленом не является. Это действительно многочлен в случае $V=\mathbb{C}^{2}$ и $I_{i}=\left\langle f_{i}\right\rangle$, где $f_{i}=0$ - уравнения неприводимых компонент $C_{i}$ (приводимой) особенности плоской кривой $(C, 0) \subset\left(\mathbb{C}^{2}, 0\right), C=\bigcup_{i=1}^{r} C_{i}, r>1$. В этом случае $\Delta_{\left\{I_{i}\right\}}\left(t_{1}, \ldots, t_{r}\right)$ совпадает с классическим многочленом Александера от нескольких переменных алгебраического зацепления $C \cap S_{\varepsilon}^{3} \subset S_{\varepsilon}^{3}\left(S_{\varepsilon}^{3}-\right.$ сфера маленького радиуса $\varepsilon$ с центром в начале координат в $\mathbb{C}^{2}$ ), см. [9]. Мы предпочитаем сохранить название «многочлен Александера» в общем случае.

Пусть $(V, 0)$ - росток аналитического пространства, и пусть $\mathbb{P} \mathcal{O}_{V, 0}$ - проективизация кольца $\mathcal{O}_{V, 0}$ ростков функций на $(V, 0)$. Следующее понятие интеграла по отношению к эйлеровой характеристике по пространству $\mathbb{P} \mathcal{O}_{V, 0}$ было описано, например, в [3].

Пусть $J_{V, 0}^{k}=\mathcal{O}_{V, 0} / \mathfrak{m}^{k+1}-$ пространство $k$-струй функций на $(V, 0)(\mathfrak{m}-$ максимальный идеал в кольце $\left.\mathcal{O}_{V, 0}\right)$ и $\mathbb{P} J_{V, 0}^{k}-$ его проективизация, $\mathbb{P}^{*} J_{V, 0}^{k}:=$ $\mathbb{P} J_{V, 0}^{k} \cup\{*\}$. Пусть $\pi_{k}: \mathbb{P} \mathcal{O}_{V, 0} \rightarrow \mathbb{P}^{*} J_{V, 0}^{k}$ - естественное отображение. Подмножество $A \subset \mathbb{P} \mathcal{O}_{V, 0}$ называется иилиндрическим, если $A=\pi_{k}^{-1}(B)$ для конструктивного подмножества $B \subset \mathbb{P} J_{V, 0}^{k} \subset \mathbb{P}^{*} J_{V, 0}^{k}$. Эйлерова характеристика $\chi(A)$ цилиндрического множества $A=\pi_{k}^{-1}(B) \subset \mathbb{P O}_{V, 0}$ определяется как эйлерова 
характеристика множества $B$. Функция $\psi: \mathbb{P O}_{V, 0} \rightarrow G$ со значениями в абелевой группе $G$ называется иилиндрической, если для любого $a \in G, a \neq 0$, множество $\psi^{-1}(a)$ цилиндрично. Интеграл $\int_{\mathbb{P} \mathcal{O}_{V, 0}} \psi d \chi$ цилиндрической функции $\psi$ по пространству $\mathbb{P} \mathcal{O}_{V, 0}$ по отношению к эйлеровой характеристике - это сумма $\sum_{a \in G \backslash\{0\}} \chi\left(\psi^{-1}(a)\right) a$ (если она имеет смысл в группе $\left.G\right)$.

Пусть

$$
\mathcal{O}_{V, 0}=J(0) \supset J(1) \supset J(2) \supset \cdots
$$

- фильтрация кольца $\mathcal{O}_{V, 0} \mathbb{C}^{*}$-инвариантными подмножествами $J(k)$ (не обязательно векторными подпространствами) с цилиндрическими проективизациями $\mathbb{P} J(k) \backslash \mathbb{P} J(k+1) \subset \mathbb{P} \mathcal{O}_{V, 0}$.

Определение. Ряд Пуанкаре фильтрации (1) - это ряд

$$
P(t)=\sum_{k=0}^{\infty} \chi(\mathbb{P} J(k) \backslash \mathbb{P} J(k+1)) \cdot t^{k} \in \mathbb{Z}[[t]] .
$$

Фильтрация вида (1) может быть определена соответствующей ( $\mathbb{C}^{*}$-инвариантной) тавтологической функцией $v: \mathcal{O}_{V, 0} \rightarrow \mathbb{Z}_{\geqslant 0} \cup\{+\infty\}: v(g)=\sup \{k: g \in$ $J(k)\}, J(k)=\left\{g \in \mathcal{O}_{V, 0}: v(g) \geqslant k\right\}$. Функция $v$ является цилиндрической в том смысле, что для любого $k \in \mathbb{Z}_{\geqslant 0}$ множество $\mathbb{P} v^{-1}(k)=\mathbb{P} J(k) \backslash \mathbb{P} J(k+1)$ цилиндрично.

Определение (2) может быть переписано в виде $P(t)=\int_{\mathbb{P O}_{V, 0}} t^{v(g)} d \chi$, где $t^{\infty}$ предполагается равным нулю.

Пример. Если все элементы $J(k)$ фильтрации являются векторными подпространствами пространства $\mathcal{O}_{V, 0}$, то

$$
P(t)=\sum_{k=0}^{\infty} \operatorname{dim}(J(k) \backslash J(k+1)) \cdot t^{k} .
$$

Пусть $\left\{v_{1}, \ldots, v_{r}\right\}$ - набор $\mathbb{C}^{*}$-инвариантных цилиндрических функций на $\mathcal{O}_{V, 0}$ со значениями в $\mathbb{Z}_{\geqslant 0} \cup\{+\infty\}$. Этот набор определяет мультииндексную фильтрацию на $\mathcal{O}_{V, 0}:$ для $\underline{v}=\left(v_{1}, \ldots, v_{r}\right) \in \mathbb{Z}_{\geqslant 0}^{r}$

$$
J(\underline{v})=\left\{g \in \mathcal{O}_{V, 0}: \underline{v}(g) \geqslant \underline{v}\right\}
$$

$\left(\underline{v}(g):=\left(v_{1}(g), \ldots, v_{r}(g)\right), \underline{v}^{\prime} \geqslant \underline{v}^{\prime \prime}\right.$ тогда и только тогда, когда $v_{i}^{\prime} \geqslant v_{i}^{\prime \prime}$ для всех $i$ ). Множества $J(\underline{v})$, вообще говоря, не являются векторными подпространствами пространства $\mathcal{O}_{V, 0}$. Множества $\mathbb{P} J(\underline{v}) \backslash \bigcup_{i=1}^{r} \mathbb{P} J\left(\underline{v}+\underline{1}_{i}\right)$, где $\underline{1}_{i}:=$ $(0, \ldots, 0,1,0, \ldots, 0)$ (1 находится на $i$-м месте), являются цилиндрическими.

Определение. Ряд Пуанкаре фильтрации (3) - это ряд

$$
\begin{aligned}
P\left(t_{1}, \ldots, t_{r}\right) & =\int_{\mathbb{P O}_{V, 0}} \underline{\underline{t}}^{\underline{(g)}} d \chi \\
& =\sum_{\underline{v} \in \mathbb{Z}_{\geqslant 0}^{r}} \chi\left(\mathbb{P} J(\underline{v}) \backslash \bigcup_{i=1}^{r} \mathbb{P} J\left(\underline{v}+\underline{1}_{i}\right)\right) \cdot \underline{t}^{\underline{v}} \in \mathbb{Z}\left[\left[t_{1}, \ldots, t_{r}\right]\right],
\end{aligned}
$$

где $\underline{t} \underline{v}:=t_{1}^{v_{1}} \cdots t_{r}^{v_{r}}$. 
Пример. Если все $v_{i}, i=1, \ldots, r$, являются функииями порядка, т.е. $v_{i}\left(g_{1}+g_{2}\right) \geqslant \min \left\{v_{i}\left(g_{1}\right), v_{i}\left(g_{2}\right)\right\}$, то все элементы $J(\underline{v})$ фильтрации являются векторными подпространствами пространства $\mathcal{O}_{V, 0}$ и определение (4) ряда Пуанкаре становится эквивалентным определению в [2], [3], данному в терминах размерностей.

Пусть теперь $(S, 0)$ - росток нормальной особенности поверхности. Для ростка функции $g \in \mathcal{O}_{S, 0}$ и для дивизора $\gamma$ на $(S, 0)$ определено число пересечения $(\gamma \circ g) \in \mathbb{Z} \cup\{\infty\}$. Его можно определить, например, следующим образом. Пусть $\gamma=\sum_{i} k_{i} \gamma_{i}$, где $\gamma_{i}$ - дивизоры, представленные неприводимыми кривыми $\left(C_{i}, 0\right)$ на $(S, 0)$. Тогда $(\gamma \circ g)=\sum_{i} k_{i}\left(\gamma_{i} \circ g\right)$, где $\left(\gamma_{i} \circ g\right)-$ порядок нуля функции $g$ на кривой $\left(C_{i}, 0\right)$ в униформизирующем параметре. (Если $\left.g\right|_{C_{i}} \equiv 0$, то $\left(\gamma_{i} \circ g\right)$ предполагается равным $+\infty$.) Если $\gamma$ - дивизор Картье, $\gamma=\{f=0\}$, $f \in \mathcal{O}_{S, 0}$, мы будем писать $(f \circ g)$ вместо $(\gamma \circ g)$.

Пусть $I$ - идеал в кольце $\mathcal{O}_{S, 0}$, и пусть $v_{I}(g):=\min \{(g \circ f): f \in I\}$. Можно убедиться, что функция $v_{I}: \mathcal{O}_{S, 0} \rightarrow \mathbb{Z}_{\geqslant 0} \cup\{\infty\}$ является $\mathbb{C}^{*}$-инвариантной и цилиндрической.

Определение. Фильтрачия, соответствующая идеалу I, - это фильтрация

$$
\mathcal{O}_{S, 0}=J_{I}(0) \supset J_{I}(1) \supset J_{I}(2) \supset \ldots,
$$

определенная функцией $v_{I}$ :

$$
J_{I}(k)=\left\{g \in \mathcal{O}_{S, 0}: v_{I}(g) \geqslant k\right\} .
$$

Пусть теперь $\left\{I_{1}, \ldots, I_{r}\right\}$ - набор идеалов в кольце $\mathcal{O}_{S, 0}$.

Определение. Мультииндексная фильтрация, соответствующая набору идеалов $\left\{I_{1}, \ldots, I_{r}\right\}$, - это фильтрация кольца $\mathcal{O}_{S, 0}$, элементами которой являются

$$
J_{\left\{I_{i}\right\}}(\underline{v})=\bigcap_{i=1}^{r} J_{I_{i}}\left(v_{i}\right)
$$

$\left(\underline{v}=\left(v_{1}, \ldots, v_{r}\right) \in \mathbb{Z}_{\geqslant 0}^{r}\right)$.

Эта фильтрация определяется набором $\left\{v_{I_{i}}\right\}$ соответствующих функций. Пространства $J_{\left\{I_{i}\right\}}(\underline{v})$ не являются, вообще говоря, векторными подпространствами в $\mathcal{O}_{S, 0}$. Можно увидеть, что подмножества $J_{\left\{I_{i}\right\}}(\underline{v})$ являются так называемыми «линейными подпространствами», т. е. принадлежат алгебре множеств, порожденной векторными подпространствами.

Примеры. 1. Пусть $(C, 0) \subset(S, 0)$ - росток (приведенной) кривой на поверхности $(S, 0), C=\bigcup_{i=1}^{r} C_{i}$ - разложение кривой $C$ на неприводимые компоненты, и пусть $I_{C_{i}}$ - идеал кривой $C_{i}$. Имеется мультииндексная фильтрация, соответствующая набору идеалов $\left\{I_{C_{1}}, \ldots, I_{C_{r}}\right\}$. Это фильтрация кольца $\mathcal{O}_{S, 0}$ идеалами. Если $S=\mathbb{C}^{2}$ или если все компоненты $C_{i}$ кривой $C$ являются дивизорами Картье на $(S, 0)$ (это так, например, для любой кривой на рациональной двойной точке типа $E_{8}$ ), она совпадает с фильтрацией, соответствующей кривой $C$, которая рассмотрена в [2] и [5]. В противном случае это, вообще говоря, не так. Для $S=\mathbb{C}^{2}$ ряд Пуанкаре этой фильтрации совпадает с многочленом Александера (от $r$ переменных) кривой $(C, 0)$, т. е. соответствующего зацепления $C \cap S_{\varepsilon}^{3} \subset S_{\varepsilon}^{3}[2]$. 
2. Пусть $\pi:(X, D) \rightarrow(S, 0)$ - собственная модификация поверхности $(S, 0)$, которая является изоморфизмом вне нуля, такая, что $X$ неособо, а $D=\pi^{-1}(0)$ - дивизор с нормальными пересечениями на $X$. Пусть $D=\bigcup_{\sigma \in \Gamma} E_{\sigma}$ - представление исключительного дивизора $D$ в виде объединения его неприводимых компонент. Для $\sigma \in \Gamma$, т. е. для компоненты $E_{\sigma}$ исключительного дивизора $D$, пусть $\widetilde{L}$ - росток гладкой неприводимой кривой на $X$, пересекающий $E_{\sigma}$ трансверсально в неособой точке дивизора $D$ (т. е. не в точке пересечения компоненты $E_{\sigma}$ с другой компонентой исключительного дивизора $\left.D\right), L=\pi(\widetilde{L})-$ соответствующая кривая на $(S, 0), I_{L} \subset \mathcal{O}_{S, 0}$ - идеал кривой $(L, 0)$ и $I_{E_{\sigma}}-$ идеал, порожденный всеми идеалами $I_{L}$ описанного вида. Для $r$ выбранных компонент $E_{1}, \ldots, E_{r}$ исключительного дивизора $D$, т. е. для $\{1, \ldots, r\} \subset \Gamma$, таким образом получается мультииндексная фильтрация (идеалами), соответствующая набору $\left\{E_{1}, \ldots, E_{r}\right\}$ компонент исключительного дивизора $D$. Опять если $S=\mathbb{C}^{2}$ или если все описанные выше кривые $L$ являются дивизорами Картье на $(S, 0)$, эта фильтрация совпадает с дивизориальной фильтрацией, изучавшейся в [8] и [4]. В противном случае это, вообще говоря, не так. Для $S=\mathbb{C}^{2}$ ряд Пуанкаре этой фильтрации совпадает с многочленом Александера набора идеалов $\left\{I_{E_{1}}, \ldots, I_{E_{r}}\right\}$ [8].

3. Можно рассмотреть набор нормирований $v_{1}, \ldots, v_{r^{\prime}}, w_{1}, \ldots, w_{r^{\prime \prime}}$, соответствующих компонентам $E_{1}, \ldots, E_{r^{\prime}}$ модификации $\pi:(X, D) \rightarrow(S, 0)$ и неприводимым росткам кривых $\left(C_{i}, 0\right) \subset(S, 0), i=1, \ldots, r^{\prime \prime}$, соответственно. Если $S=\mathbb{C}^{2}$, а модификация $\pi$ является относительным (embedded) разрешением кривой $C=\bigcup_{i=1}^{r^{\prime \prime}} C_{i}$, то имеет место следующая формула для ряда Пуанкаре соответствующей мультииндексной фильтрации (идеалами). Пусть $\left(m_{\sigma \delta}\right)-$ минус обратная матрица к матрице пересечений $\left(E_{\sigma} \circ E_{\delta}\right)$ компонент $E_{\sigma}$ на $X, \alpha_{i} \in \Gamma, i=1, \ldots, r^{\prime \prime}$, - номер компоненты $E_{\alpha_{i}}$ исключительного дивизора $D$, пересекающей собственный прообраз (неприводимой) кривой $C_{i}$, и $\underline{m}_{\sigma}^{\prime}:=$ $\left(m_{\sigma 1}, \ldots, m_{\sigma r^{\prime}}\right), \underline{m}_{\sigma}^{\prime \prime}:=\left(m_{\sigma \alpha_{1}}, \ldots, m_{\sigma \alpha_{r^{\prime \prime}}}\right)$. Пусть $\stackrel{\circ}{E}_{\sigma}-$ неособая часть компоненты $E_{\sigma}$ в объединении полных прообразов кривых $C_{i}$, т. е. $E_{\sigma}$ минус точки пересечения с другими компонентами исключительного дивизора $D$ и с собственными прообразами кривых $C_{i}, i=1, \ldots, r^{\prime \prime}$. Как в [3] и [8] (см. также доказательство теоремы 1), можно показать, что

$$
P\left(t_{1}, \ldots, t_{r^{\prime}}, T_{1}, \ldots, T_{r^{\prime \prime}}\right)=\prod_{\sigma \in \Gamma}\left(1-\underline{t}^{\underline{m^{\prime}}} \underline{T}^{\underline{m}^{\prime \prime}}\right)^{-\chi\left(\stackrel{\circ}{E_{\sigma}}\right)}
$$

(здесь $\underline{t}=\left(t_{1}, \ldots, t_{r^{\prime}}\right)$ и $\underline{T}=\left(T_{1}, \ldots, T_{r^{\prime \prime}}\right)-$ переменные, соответствующие нормированиям $v_{1}, \ldots, v_{r^{\prime}}$ и $w_{1}, \ldots, w_{r^{\prime \prime}}$ соответственно).

Пусть $\left\{I_{1}, \ldots, I_{r}\right\}$ - набор идеалов в кольце $\mathcal{O}_{\mathbb{C}^{2}, 0}$ и $\pi:(X, D) \rightarrow\left(\mathbb{C}^{2}, 0\right)-$ разрешение этого набора идеалов. Пусть $D=\bigcup_{\sigma \in \Gamma} E_{\sigma}$, где $E_{\sigma}-$ неприводимые компоненты исключительного дивизора $D$ (каждая компонента $E_{\sigma}$ изоморфна комплексной проективной прямой $\left.\mathbb{C P}^{1}\right)$. Для $\sigma \in \Gamma$, т.е. для компоненты $E_{\sigma}$ исключительного дивизора $D$, пусть $\stackrel{\circ}{E_{\sigma}}-$ «неособая часть» компоненты $E_{\sigma}$ в объединении множеств нулей идеалов $\left\{I_{i}^{*}\right\}$, т. е. $E_{\sigma}$ минус точки пересечения со всеми другими компонентами объединения. Пусть $k_{\sigma i}-$ кратность компоненты $E_{\sigma}$ в дивизоре нулей идеала $I_{i}$, и пусть $\underline{k}_{\sigma}:=\left(k_{\sigma 1}, \ldots, k_{\sigma r}\right) \in \mathbb{Z}_{\geqslant 0}^{r}$. 


\section{Теорема 1. Имеет место равенство}

$$
P_{\left\{I_{i}\right\}}\left(t_{1}, \ldots, t_{r}\right)=\prod_{\sigma \in \Gamma}\left(1-\underline{t}^{\underline{k}}\right)^{-\chi\left(\stackrel{\circ}{E}_{\sigma}\right)} .
$$

Это утверждение обобщает утверждения из [2] и [8] для идеалов, описанных в примерах 1 и 2 (для $S=\mathbb{C}^{2}$ ). Можно видеть, что правая часть равенства (5) равна многочлену Александера $\Delta_{\left\{I_{i}\right\}}\left(t_{1}, \ldots, t_{r}\right)$ набора идеалов $\left\{I_{i}\right\}$ и, таким образом, в этом случае ряд Пуанкаре совпадает с многочленом Александера.

Доказательство теоремы 1. Доказательство по существу повторяет рассуждения из [3], [8]. Используется представление ряда Пуанкаре в виде интеграла по отношению к эйлеровой характеристике. Имеется отображение из проективизации $\mathbb{P} \mathcal{O}_{\mathbb{C}^{2}, 0}$ кольца $\mathcal{O}_{\mathbb{C}^{2}, 0}$ на пространство эффективных дивизоров на $\stackrel{\circ}{D}=\bigcup_{\sigma \in \Gamma} \stackrel{\circ}{E_{\sigma}}$ : ростку функции $f$ на $\left(\mathbb{C}^{2}, 0\right)$ сопоставляется пересечение собственного прообраза кривой $\{f=0\}$ с исключительным дивизором $D$. (Для наперед заданного $N$ это отображение будет определено для всех ростков функций $f$ с $v_{I_{i}}(f) \leqslant N, i=1, \ldots, r$, если сделать достаточно много дополнительных сигма-процессов в точках пересечения компонент объединения множеств нулей идеалов $I_{i}^{*}$ на $X$. Неособые части $E_{\sigma}$ всех дополнительных компонент $E_{\sigma}$ исключительного дивизора $D$ изоморфны комплексной проективной прямой $\mathbb{C} \mathbb{P}^{1}$ без двух точек. Поэтому их эйлеровы характеристики равны нулю и они не вносят вклад в правую часть равенства (5).) Из предложения 2 работы [3] следует, что прообраз точки при этом отображении является комплексным аффинным пространством и поэтому имеет эйлерову характеристику, равную 1 . Из формулы Фубини следует, что ряд Пуанкаре $P_{\left\{I_{i}\right\}}\left(t_{1}, \ldots, t_{r}\right)$ равен интегралу по отношению к эйлеровой характеристике монома $\underline{t} \underline{v}$ по пространству эффективных дивизоров на $\stackrel{\circ}{D}$. Здесь $\underline{v}$ - аддитивная функция на пространстве эффективных дивизоров на $\stackrel{\circ}{D}$ (со значениями в $\mathbb{Z}_{\geqslant 0}^{r}$ ), равная $\underline{k}_{\sigma}$ для точки из компоненты ${\stackrel{\circ}{E_{\sigma}}}$.

Пространство эффективных дивизоров на $\stackrel{\circ}{D}$ является прямым произведением пространств эффективных дивизоров на компонентах $\stackrel{\circ}{E}_{\sigma}, \sigma \in \Gamma$. Каждое из этих пространств является несвязным объединением симметрических степеней $S^{\ell} \stackrel{\circ}{E}_{\sigma}$ компонент $\stackrel{\circ}{E_{\sigma}}$. Поэтому

$$
P_{\left\{I_{i}\right\}}\left(t_{1}, \ldots, t_{r}\right)=\prod_{\sigma \in \Gamma}\left(\sum_{\ell=0}^{\infty} \chi\left(S^{\ell} \stackrel{\circ}{E}_{\sigma}\right) \cdot \underline{t}^{\ell \underline{k}}\right) .
$$

Теперь равенство (5) следует из формулы $\sum_{\ell=0}^{\infty} \chi\left(S^{\ell} X\right) t^{\ell}=(1-t)^{-\chi(X)}$.

Пример. По определению многочлен Александера набора идеалов $\left\{I_{1}, \ldots\right.$, $\left.I_{r}\right\}$ в кольце $\mathcal{O}_{\mathbb{C}^{2}, 0}$ совпадает с многочленом Александера набора их целых замыканий $\left\{\bar{I}_{1}, \ldots, \bar{I}_{r}\right\}$. Целозамкнутый идеал $I=\bar{I}$ можно представить в виде $I=\prod_{\sigma} I_{E_{\sigma}}^{n_{\sigma}} \prod_{j=1}^{r^{\prime \prime}} I_{C_{j}}^{m_{j}}$, где $I_{E_{\sigma}}$ - идеал, соответствующий дивизору $E_{\sigma}$ разрешения идеала $I$, а $I_{C_{j}}$ - идеал неприводимой особенности кривой $\left(C_{j}, 0\right) \subset\left(\mathbb{C}^{2}, 0\right)$, $m_{j}>0$ (см. [16]). Пусть $s=\# \Gamma-$ количество компонент исключительного дивизора $D$ разрешения. Имеет место равенство $n_{\sigma}=\sum_{\delta \in \Gamma} m_{\sigma \delta} k_{\delta}$, где $k_{\delta}$ - кратность идеала $I$ на компоненте $E_{\delta}$ исключительного дивизора $D$, а 
$\left(m_{\sigma \delta}\right)$ - минус обратная матрица к матрице пересечений $\left(E_{\sigma} \circ E_{\delta}\right)$ компонент $E_{\sigma}$ на $X$. Поэтому имеет место следующая формула для функции порядка $v_{I}: \mathcal{O}_{\mathbb{C}^{2}, 0} \backslash\{0\} \rightarrow \mathbb{Z}_{\geqslant 0}^{r} \cup\{\infty\}:$

$$
v_{I}=\sum_{\sigma} n_{\sigma} v_{\sigma}+\sum_{j=1}^{r^{\prime \prime}} m_{j} w_{j}=\sum_{\sigma, \delta} m_{\sigma \delta} k_{\delta} v_{\sigma}+\sum_{j=1}^{r^{\prime \prime}} m_{j} w_{j},
$$

где $v_{\sigma}$ - функция порядка, соответствующая компоненте $E_{\sigma}$ (пример 2 на с. 44), $w_{j}$ - функция порядка, соответствующая неприводимой кривой $\left(C_{j}, 0\right)($ пример 1 на с. 43). Имеет место следующая формула:

$$
P_{I}(t)=P\left(t^{n_{1}}, \ldots, t^{n_{s}}, t^{m_{1}}, \ldots, t^{m_{r^{\prime \prime}}}\right),
$$

где $P\left(t_{1}, \ldots, t_{s}, T_{1}, \ldots, T_{r^{\prime \prime}}\right)$ - ряд Пуанкаре набора нормирований $v_{1}, \ldots, v_{s}$, $w_{1}, \ldots, w_{r^{\prime \prime}}$, соответствующих компонентам $E_{\sigma}$ исключительного дивизора и кривым $\left(C_{j}, 0\right)$ (пример 3 на с. 44$)$. При этом имеет место следующее утверждение.

Предложение 1. Пусть $\left(I_{1}, \ldots, I_{r}\right)$ - набор идеалов в $\mathcal{O}_{\mathbb{C}^{2}, 0}$, u nусть $I_{i}=$ $\prod_{\sigma} I_{\sigma}^{n_{\sigma}^{i}} \prod_{j=1}^{r^{\prime \prime}} I_{C_{j}}^{m_{j}^{i}}, i=1, \ldots, r$, где для любого $j=1, \ldots, r^{\prime \prime}$ существует $i$, такое что $m_{j}^{i}>0$. Тогда

$$
P_{\left\{I_{i}\right\}}\left(t_{1}, \ldots, t_{r}\right)=P\left(\prod_{i=1}^{r} t_{i}^{n_{1}^{i}}, \ldots, \prod_{i=1}^{r} t_{i}^{n_{s}^{i}}, \prod_{i=1}^{r} t_{i}^{m_{1}^{i}}, \ldots, \prod_{i=1}^{r} t_{i}^{m_{r^{\prime \prime}}^{i}}\right) .
$$

Аналогичным образом можно доказать версии основных утверждений из [4] и [7] для идеалов в кольце функций на рациональной особенности поверхности или на ее универсальном абелевом накрытии. Пусть $(S, 0)$ - рациональная особенность поверхности, и пусть $\pi:(X, D) \rightarrow(S, 0)$ - ее разрешение. Зацепление $S \cap S_{\varepsilon}^{3}$ особенности $(S, 0)$ является рациональной гомологической сферой, и ее первая группа гомологий $H=H_{1}(S \backslash\{0\})$ изоморфна коядру $\mathbb{Z}^{\Gamma} / \operatorname{Im} j$ отображения $j: \mathbb{Z}^{\Gamma} \rightarrow \mathbb{Z}^{\Gamma}$, определенного матрицей пересечений $\left(E_{\sigma} \circ E_{\sigma^{\prime}}\right)$ (порядок группы $H$ равен определителю $d$ минус матрицы пересечений $\left.-\left(E_{\sigma} \circ E_{\sigma^{\prime}}\right)\right)$. Для $\sigma \in \Gamma$ пусть $h_{\sigma}$ - элемент группы $H$, представленный петлей в многообразии $X \backslash D \simeq S \backslash\{0\}$, обходящей вокруг компоненты $E_{\sigma}$ в положительном направлении. Группа $H$ порождена элементами $h_{\sigma}$ для всех $\sigma \in \Gamma$.

Пусть $p:(\widetilde{S}, 0) \rightarrow(S ; 0)$ - универсальное абелево накрытие особенности поверхности $(S, 0)$ (см., например, [12], [13], [11]). Группа $H$ действует на $(\widetilde{S}, 0)$, и ограничение $\left.p\right|_{\widetilde{S} \backslash\{0\}}$ отображения $p$ на дополнение к началу координат является (обычным, неразветвленным) накрытием $\widetilde{S} \backslash\{0\} \rightarrow S \backslash\{0\}$ со структурной группой $H$. Можно поднять отображение $p$ на (разветвленное) накрытие $p^{\prime}:(\widetilde{X}, \widetilde{D}) \rightarrow(X, D)$, где $\widetilde{X}-$ нормальная поверхность (вообще говоря, не гладкая) и $\widetilde{X} \backslash \widetilde{D} \simeq \widetilde{S} \backslash\{0\}:$

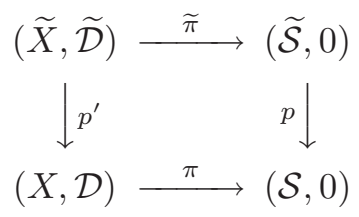


(можно определить $\widetilde{X}$ как нормализацию расслоенного произведения $X \times \mathcal{S} \widetilde{\mathcal{S}}$ многообразий $X$ и $\widetilde{\mathcal{S}}$ над $\mathcal{S})$.

Пусть $R(H)$ - кольцо (виртуальных) представлений группы $H$. Для $\sigma \in \Gamma$, т. е. для компоненты $E_{\sigma}$ исключительного дивизора $D$, пусть $\alpha_{\sigma}-$ одномерное представление $H \rightarrow \mathbb{C}^{*}=\mathbf{G L}(1, \mathbb{C})$ группы $H$, определенное формулой $\alpha_{\sigma}\left(h_{\delta}\right)=\exp \left(-2 \pi \sqrt{-1} m_{\sigma \delta}\right)$ (здесь знак минус отражает тот факт, что действие элемента $h \in H$ на кольце $\mathcal{O}_{\widetilde{\mathcal{S}}, 0}$ определяется формулой $\left.(h \cdot f)(x)=f\left(h^{-1}(x)\right)\right)$. Пусть $\left\{I_{1}, \ldots, I_{r}\right\}$ - набор идеалов в $\mathcal{O}_{S, 0}$, и пусть $\widetilde{I}_{i}=p^{*} I_{i}$ - поднятия идеалов $I_{i}$ на универсальное абелево накрытие $\widetilde{S}$. Соответствующая мультииндексная фильтрация на кольце $\mathcal{O}_{\widetilde{S}, 0}$ ростков функций на абелевом накрытии $\widetilde{S}$ является $H$-инвариантной. Понятие эквивариантного ряда Пуанкаре такой мультииндексной фильтрации было определено в [6]. Аналогично [7] получается следующий результат.

Теорема 2. Если разрешение $\pi:(X, D) \rightarrow(S, 0)$ является разрешением набора идеалов $\left\{I_{1}, \ldots, I_{r}\right\}$, имеет место формула

$$
P^{H}\left(t_{1}, \ldots, t_{r}\right)=\prod_{\sigma \in \Gamma}\left(1-\alpha_{\sigma} \underline{t}^{d \underline{k} \sigma}\right)^{-\chi\left(\stackrel{\circ}{E}_{\sigma}\right)} .
$$

Сумма мономов этого ряда с тривиальным представлением в коэффициентах (т. е. тех мономов, для которых $\sum_{\sigma} m_{\delta \sigma} v_{\sigma}$ является целым числом для любого $\delta \in \Gamma)$ с заменой переменных $t_{i}^{d} \rightarrow t_{i}$ является рядом Пуанкаре фильтрации на $\mathcal{O}_{S, 0}$, соответствующей набору идеалов $\left\{I_{i}\right\}$ (ср. с [4], [5]).

Замечание. Целозамкнутые идеалы в кольце $\mathcal{O}_{S, 0}$ ростков функций на рациональной особенности поверхности $(S, 0)$ имеют описание, подобное тому, которое дано в примере перед предложением 1 для $S=\mathbb{C}^{2}$. Пусть $I=\bar{I}-$ целозамкнутый идеал в $\mathcal{O}_{S, 0}$, и пусть $\pi:(X, 0) \rightarrow(S, 0)$ - его разрешение. Для $\sigma \in \Gamma$, т. е. для компоненты $E_{\sigma}$ исключительного дивизора $D$, пусть $\widetilde{L}-$ росток неособой неприводимой кривой на $X$, пересекающий $E_{\sigma}$ трансверсально в неособой точке, т. е. в точке из $\stackrel{\circ}{E_{\sigma}}$, и пусть $L=\pi(\widetilde{L})$. Существует наименьшее натуральное число $d_{\sigma}$, такое, что $d_{\sigma} L$ - дивизор Картье на $(S, 0)$ : $d_{\sigma} L=\left(g_{L}\right)$ для $g_{L} \in \mathcal{O}_{S, 0}$. (Число $d_{\sigma}$ является наименьшим натуральным числом, таким, что $d_{\sigma} m_{\sigma \delta}$ целые для всех $\delta \in \Gamma$, и равно порядку элемента $h_{\sigma}$ в группе $H=H_{1}(S \backslash\{0\})$.) Пусть $I_{\sigma}^{\prime} \subset \mathcal{O}_{S, 0}$ - идеал, порожденный всеми ростками $g_{L}$ описанного вида.

Целозамкнутый идеал $I$ имеет единственное представление вида $I=\prod_{\sigma} I_{\sigma}^{r_{\sigma}}$ для неотрицательных рациональных чисел $r_{\sigma}$, таких, что $\sum_{\sigma}\left(E_{\sigma} \circ E_{\delta}\right) r_{\sigma}$ целые для всех $\delta \in \Gamma$ (см. [10]). (Если $I_{1}$ и $I_{2}$ - целозамкнутые идеалы в кольце $\mathcal{O}_{S, 0}$, то пишут, что $I_{1}=I_{2}^{1 / q}$, если $I_{1}^{q}=I_{2}$. Если такой идеал $I_{1}$ для идеала $I_{2}$ существует, он определен однозначно.) Как уже упоминалось, дивизориальная фильтрация на кольце $\mathcal{O}_{S, 0}$, соответствующая компоненте $E_{\sigma}$, не является, вообще говоря, фильтрацией, соответствующей некоторому идеалу. Однако для соответствующих функций имеет место равенство $v_{I_{\sigma}^{\prime}}=d_{\sigma} v_{E_{\sigma}}$. Поэтому ряд Пуанкаре фильтрации, соответствующей идеалу $I_{\sigma}^{\prime}$, получается из ряда Пуанкаре дивизориальной фильтрации, соответствующей компоненте $E_{\sigma}$, заменой 
переменной $t$ на $t^{d_{\sigma}}$. Это объясняет соотношение между рядом Пуанкаре фильтрации на кольце $\mathcal{O}_{S, 0}$, соответствующей набору идеалов, и рядом Пуанкаре дивизориальной фильтрации, как в примере перед предложением 1.

\section{ЛитературА}

[1] N. A'Campo, La fonction zêta d'une monodromie, Comm. Math. Helv., 50 (1975), 233-248.

[2] A. Campillo, F. Delgado, S. M. Gusein-Zade, The Alexander polynomial of a plane curve singularity via the ring of functions on it, Duke Math. J., 117:1 (2003), 125156.

[3] A. Campillo, F. Delgado, S. M. Gusein-Zade, The Alexander polynomial of a plane curve singularity and integrals with respect to the Euler characteristic, Internat. J. Math., 14:1 (2003), 47-54.

[4] A. Campillo, F. Delgado, S. M. Gusein-Zade, Poincaré series of a rational surface singularity, Invent. Math., 155:1 (2004), 41-53.

[5] A. Campillo, F. Delgado, S. M. Gusein-Zade, Poincaré series of curves on rational surface singularities, Comm. Math. Helv., 80:1 (2005), 95-102.

[6] A. Campillo, F. Delgado, S. M. Gusein-Zade, On Poincaré series of filtrations on equivariant functions of two variables, Mosc. Math. J., 7:2 (2007), 243-255.

[7] С. М. Гусейн-Заде, Ф. Дельгадо, А. Кампильо, Универсальные абелевы накрытия рациональных особенностей поверхностей и мультииндексные фильтрации, Функц. анализ и его прил., 42:2 (2008), 3-10.

[8] F. Delgado, S. M. Gusein-Zade, Poincaré series for several plane divisorial valuations, Proc. Edinb. Math. Soc. (2), 46:2 (2003), 501-509.

[9] D. Eisenbud, W. Neumann, Three-Dimensional Link Theory and Invariants of Plane Curve Singularities, Ann. of Math. Studies, vol. 110, Princeton Univ. Press, Princeton, NJ, 1985.

[10] J. Lipman, Rational singularities with applications to algebraic surfaces and unique factorization, Inst. Hautes Études Sci. Publ. Math., 36 (1969), 195-279.

[11] A. Némethi, Poincaré series associated with surface singularities, in: Singularities I, Contemp. Math., vol. 474, Amer. Math. Soc., Providence, RI, 2008, 271-297.

[12] W. D. Neumann, J. Wahl, Universal abelian covers of surface singularities, in: Trends in singularities, Trends Math., Birkhäuser, Basel, 2002, 181-190.

[13] T. Okuma, Universal abelian covers of rational surface singularities, J. London Math. Soc. (2), 70:2 (2004), 307-324.

[14] L. van Proeyen, W. Veys, The monodromy conjecture for zeta functions associated to ideals in dimension two, Ann. Inst. Fourier (Grenoble), 60:4 (2010), 1347-1362.

[15] C. Sabbah, Modules d'Alexander et D-modules, Duke Math. J., 60:3 (1990), 729-814.

[16] О. Зарисский, П. Самюэль, Коммутативная алгебра, т. 2, ИЛ, М., 1963.

Московский государственный университет, механико-математический факультет e-mail: sabir@mccme.ru
Поступило в редакцию 23 января 2011 г.

University of Valladolid, Dept. of Algebra, Geometry and Topology

e-mail: fdelgado@agt.uva.es

University of Valladolid, Dept. of Algebra, Geometry and Topology

e-mail: campillo@agt.uva.es 